\title{
Shrinkage measurement for holographic recording materials
}

\author{
R. Fernández ${ }^{1}$, S. Gallego* ${ }^{1,2}$, A. Márquez ${ }^{1,2}$, J. Francés ${ }^{1,2}$, V. Navarro Fuster ${ }^{1,3}$, C. Neipp ${ }^{1,2}$, M. \\ Ortuño $^{1,2}$, A. Beléndez $z^{1,2}$, I. Pascual ${ }^{1,3}$ \\ ${ }^{1}$ I.U. Fisica Aplicada a Las Ciencias Y Las Tecnologías (Spain) Apartat 99 E-03080 Alacant; ${ }^{2}$ Dept. \\ Física Enginyeria de Sistemes i Teoria del Senyal, Universitat d'Alacant (Spain) Apartat 99 E-03080 \\ Alacant; ${ }^{3}$ Dept. Óptica, Farmacologia i Anatomia, Universitat d'Alacant (Spain) Apartat 99 E-03080 \\ Alacant
}

\begin{abstract}
There is an increasing demand for new holographic recording materials. One of them are photopolymers, which are becoming a classic media in this field. Their versatility is well known and new possibilities are being created by including new components, such as nanoparticles or dispersed liquid crystal molecules in classical formulations, making them interesting for additional applications in which the thin film preparation and the structural modification have a fundamental importance. Prior to obtaining a wide commercialization of displays based on photopolymers, one of the key aspects is to achieve a complete characterization of them. In this sense, one of the main parameters to estimate and control is the shrinkage of these materials. The volume variations change the angular response of the hologram in two aspects, the angular selectivity and the maximum diffraction efficiency. One criteria for the recording material to be used in a holographic data storage application is the shrinkage, maximum of $0.5 \%$. Along this work, we compare two different methods to measure the holographic recording material shrinkage. The first one is measuring the angle of propagation for both diffracted orders \pm 1 when slanted gratings are recorded, so that an accurate value of the grating vector can be calculated. The second one is based on interference measurements at zero spatial frequency limit. We calculate the shrinkage for three different photopolymers: a polyvinyl alcohol acrylamide (PVA/AA) based photopolymer, one of the greenest photopolymers whose patent belongs to the Alicante University called Biophotopol and on the last place a holographic-dispersed liquid crystal photopolymer (H-PDLC).
\end{abstract}

Keywords: holography, holographic recording materials, diffractive optics, optical materials

\section{INTRODUCTION}

Photopolymers are very appealing recording materials because of their practical properties, self-processing capabilities and their low price. They are used in the mass production of holograms for applications related with security purposes and holographic-based memories [1] in addition to other applications [2-6].

In general, the composition of photopolymers includes one or more monomers, a photosensitive dye and an initiator and many families of photopolymers has been proposed, liquid, solid, with nanoparticles or liquid crystals dissolved in its composition, with short or large polymer chains, with dyes that are not consumed or that can be eliminated with an UV cure, etc. For each specific application, an optimized chemical composition is needed to play with the large variety of possible properties like the rate of monomer diffusion, shrinkage, polymerization rate, the absorption of the material or the length of the polymer chain [7-9].

There are some criteria for the recording material to be used in a holographic data storage application. Some of them are the material sensitivity, between of $100-1000 \mathrm{~mJ} / \mathrm{cm} 2$ to achieve full dynamic range; refractive index modulation, minimum of $5 \times 10^{-3}$; and shrinkage, maximum of $0.5 \%$ [10]. This last criterion, the shrinkage is commonly assumed to occurs after the grating formation. The assumption that one side of the material is attached to a rigid substrate is the basis of the fringe rotation model [11], this can be a reminiscence of the initial steps of the holography where silver halide emulsion was the most popular holographic recording material [12]. On the other hand, the common way of modelling the Bragg shifting in replay properties it is to assume a negligible change on the refractive index [13]. Thus, the standard

Holography: Advances and Modern Trends V, edited by Miroslav Hrabovský, John T. Sheridan, Antonio Fimia, Proc. of SPIE Vol. 10233, 102330C · (C) 2017 SPIE · CCC code: 0277-786X/17/\$18 · doi: 10.1117/12.2265180 
method to measure shrinkage is measuring where the Bragg's condition is fulfilled to achieve maximum diffraction efficiency in the reconstruction scheme.

To measure the holographic shrinkage, C. Zhao et al [14] proposed a corrected condition in the geometry of optical setup for recording volume holograms. They provide explicit equations relating the shrinkage with the variation of the spatial period of the recorded gratings, and this method has been used by some authors [15-17]. The geometrical approximation proposed by Zhao et al is shown in Fig. 1. In this figure, the fringes of the grating are represented by continuous lines and separated by a spatial period $\Lambda$. The fringes after thickness changes due to the shrinkage are represented by discontinuous lines and separated by a spatial period of $\Lambda^{\prime} ; \mathrm{d}$ and $\mathrm{d}^{\prime}$ are the initial thickness and the thickness after shrinkage respectively; $\mathrm{L}$ is the maximum length of the fringes and $\Lambda \mathrm{x}$ and $\mathrm{Lx}$ are two constants independent of shrinkage and derived from the assumed conservation of the $\mathrm{x}$ component of $\mathrm{K}$, represented by $\mathrm{Kx}$ in this model. In the $\mathrm{K}$-vector diagram, $\mathrm{K}$ is the grating vector and $\rho$ and $\sigma$ are the propagation vectors of the recording beams inside the recording material.

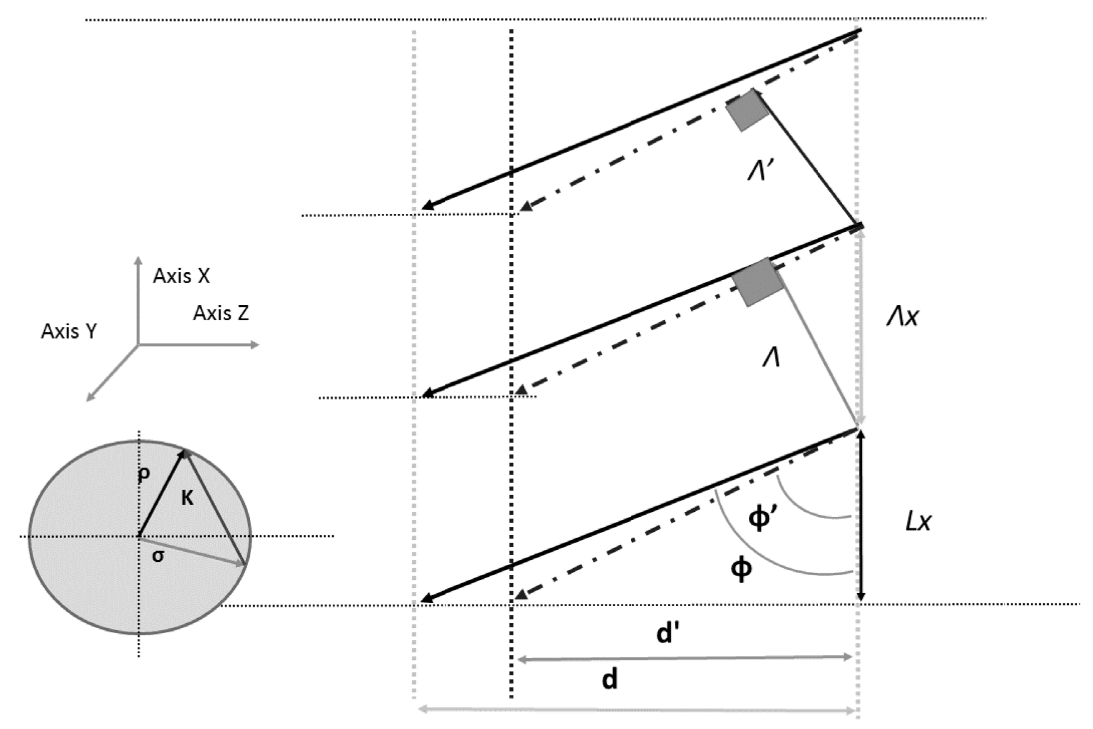

Fig. 1 Shrinkage model proposed by C. Zhao et al and K-vector diagram. Lights travels to the positive direction of axis Z.

Two expressions can be easily derived following the diagram represented in Fig 1:

$$
\begin{aligned}
& \Lambda^{\prime}=\Lambda \frac{\sin \left(\phi^{\prime}\right)}{\sin (\phi)} \\
& \mathrm{d}^{\prime}=\mathrm{d} \frac{\tan \left(\phi^{\prime}\right)}{\tan (\phi)}
\end{aligned}
$$

Alternatively, new techniques have been proposed to study the shrinkage in photopolymers [18-23] measuring the Bragg-angle detuning and obtain shrinkage information in real time. The values of shrinkage provided by each method are slightly different and in some cases, such as the presented in [20] to measure the shrinkage in real time based on holographic interferometry. The values provided, near the $3 \%$, seem to be very elevated, based on the material used and in the attenuation of the shrinkage due to the diffusion of monomer from the dark zones to the illuminated ones, given by Fick's law.

It is important to remark that the maximum shrinkage permissible in the holographic data storage materials is $0.5 \%$. Along this work, we compare two different methods to measure the holographic recording material shrinkage for three different types of materials: one of the most used photopolymers, the one based in polyvinyl alcohol acrylamide 
(PVA/AA), one of the greenest photopolymers whose patent belongs to the Alicante University called Biophotopol [24] and a holographic-dispersed liquid crystal photopolymer (H-PDLC) [25].

\section{EXPERIMENTAL}

The first photopolymer analysed, the based on PVA/AA is composed by acrylamide (AA) as polymerizable monomer, N,N'-methylene-bis-acrylamide (BMA) as crosslinking monomer, triethanolamine (TEA) as coinitiator and plasticizer, yellowish eosin (YE) as dye, polyvinyl alcohol (PVA) as binder and a small proportion of water as additional plasticizer. The composition to create samples with a physical thickness of $75 \pm 5 \mu \mathrm{m}$ on a glass substrate $(25 \mathrm{~cm} \mathrm{x} 20 \mathrm{~cm})$ is shown in Table 1. The refractive index of this material is measured before exposure using a refractometer and obtaining a value of 1.4811 .

Table 1. Composition of the liquid solution for PVA/AA based photopolymer.

\begin{tabular}{|c|c|c|c|c|}
\hline TEA (ml) & PVA (ml) (8\% w/v) & AA (gr) & BMA (gr) & YE (0.8\% w/v) (ml) \\
\hline 2.0 & 25 & 0.84 & 0.2 & 0.6 \\
\hline
\end{tabular}

The composition used for Biophotopol photopolymer is presented in Table 2. It is composed of sodium acrylate (AONa) as polymerizable monomer, triethanolamine (TEA) as coinitiator and plasticizer, sodium salt 5-riboflavin monophosphate $(\mathrm{PRF})$ as dye and polyvinyl alcohol (PVA) as binder $(\mathrm{Mw}=130000 \mathrm{u}$, hydrolysis degree $=87.7 \%)$. The composition of the photopolymer solution is deposited on a glass substrate by gravity obtaining films of $300 \pm 10 \mu \mathrm{m}$ after water evaporation. In this case, the refractive index of the "solid" layer before exposure is 1.4730 .

Table 2. Composition of the liquid solution for photopolymer AA.

\begin{tabular}{|c|c|c|c|}
\hline PVA (\% w/v) & AONa (M) & TEA (M) & PRF (M) \\
\hline 15 & 0.34 & 0.15 & $1.00 \cdot 10^{-3}$ \\
\hline
\end{tabular}

The photopolymer with liquid crystal molecules (HPDLC), uses dipentaerythritol penta/hexa-acrylate (DPHPA) with a refractive index $n=1.490$. We use the nematic liquid crystal, licristal BL036 from Merck. It is a mixture of 4cyanobiphenyls with alkyl chains of different lengths. It has an ordinary refractive index $n 0=1.5270$, and a difference between extraordinary and ordinary index $\Delta \mathrm{n}=0.2670$ [26]. There is a difference of 0.037 between the ordinary refractive index of the liquid crystal and that of the monomer. The liquid crystal concentration was set at $28 \mathrm{wt} \%$ as the starting point for component optimization and remained practically unchanged during this process. N-vinyl-2pyrrolidone (NVP) was used as crosslinker, N-phenyl glicine (NPG) as radical generator and octanoic acid (OA) as cosolvent [23]. We used ethyl eosin (YEt) as dye.

The H-PDLC prepolymer solution is made by mixing the components under red light at which the material is not sensitive. The solution is sonicated in an ultrasonic bath, deposited between two conductive ITO glass plates of $1 \mathrm{~mm}$ thick and separated using two types of glass microspheres. The microspheres were provided by Whitehouse scientific with a thickness between 15 and $20 \mu \mathrm{m}$. The composition of this material is detailed in Table 3 . For this syrup, the refractive index before polymerization is 1.5225 .

Table 3. Composition of photopolymer H-PDLC in wt $\%$

\begin{tabular}{|c|c|c|c|c|c|}
\hline DPHPA & BL036 & YEt & NPG & NVP & OA \\
\hline 48.4 & 29.2 & 0.1 & 1.5 & 16.4 & 4.4 \\
\hline
\end{tabular}


The experimental device is shown in Fig. 2. We used two different recording wavelengths depending on the material, due to the different absorption of the dyes for the different materials. For PVA/AA and H-PDLC a Nd:YAG laser tuned at a wavelength of $532 \mathrm{~nm}$ was used to record diffraction gratings by means of continuous laser exposure. In the case of Biophotopol, we use a wavelength of $488 \mathrm{~nm}$, where the dye presents good absorption and the material has higher repeatability.

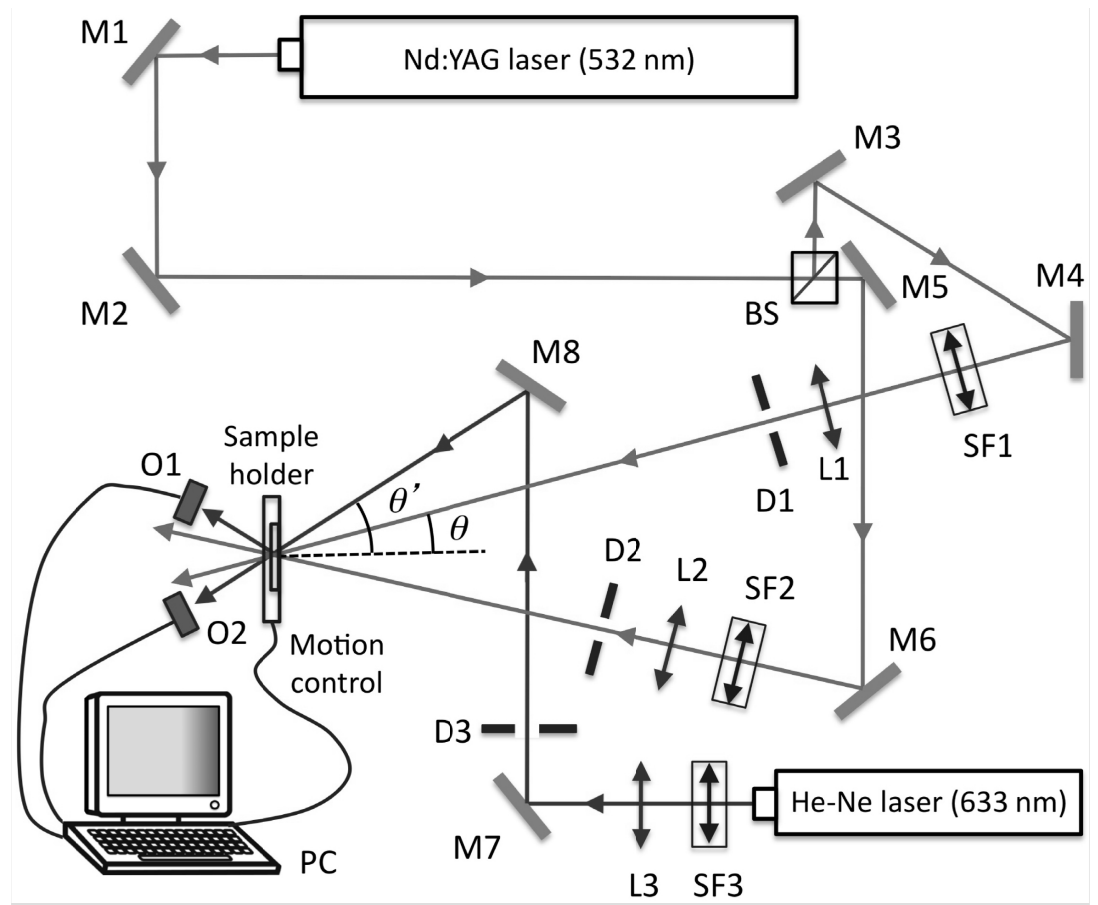

Fig. 2. Experimental set-up. BS: Beamsplitter, Mi: mirror, SFi: spatial filter, Li: lens, Di: diaphragm, Oi: optical power meter, PC: data recorder.

The object and reference beams were recombined at the sample at an angle of $15.8^{\circ}$ to the normal with an appropriate set of mirrors, and the spatial frequency obtained was 1024 lines $/ \mathrm{mm}$ for non-slanted gratings. The working intensity at 532 $\mathrm{nm}$ was $3 \mathrm{~mW} / \mathrm{cm}^{2}$, this recording intensity is the same for all the photopolymeric materials.

After recording, when the green laser was shut off, we rotated the sample to record the angular response around the different Bragg conditions, $\pm 1, \pm 2$. Fitting the angular response with different theories, we obtained the data necessary to calculate optical thickness of the holographic diffraction gratings and the value of the grating vector to calculate the shrinkage. To record slanted gratings, we rotated the sample $15^{\circ}$, that means a new spatial frequency depending on the refractive index of material. For PVA/AA with these recording and read out parameters, we obtain $1004 \mathrm{lines} / \mathrm{mm}$, and a $\mathrm{K}_{\mathrm{x}}=6.21 \mu \mathrm{m}^{-1}$. Due to the different refractive index for HPDLC we obtain just a small variation in the spatial frequency, now is 1003 lines $/ \mathrm{mm}$. In the case of Biophotopol, we use a wavelength of $488 \mathrm{~nm}$, where the dye presents good absorption and the material has higher repeatability. For symmetrical case, non-slanted, both recording beams impinge with an angle of $17.1^{\circ}$ [26], once we rotate the stage $15^{\circ}$ the spatial frequency is $1182 \mathrm{lines} / \mathrm{mm}$ and the initial value of $\mathrm{K}_{\mathrm{x}}$ is $7.314 \mu \mathrm{m}^{-1}$. For this higher spatial frequency, we expect an increase of $40-50 \%$ shrinkage value from the obtained at 1004 lines/mm as were reported in [16] for PVA/AA photopolymer.

\section{RESULTS}

The results obtained for the three different photopolymers studied are presented in this section. These results are separated in subsections per the analysis carried out: in the first place, we analysed the conservation of the x component of $\mathrm{K}$ vector, $\mathrm{K}_{\mathrm{x}}$. Secondly, we calculated the shrinkage for the different photopolymers. On the last place, we compared 
different electromagnetic methods to fit the angular responses of the diffracted orders coming out of the gratings, obtaining the optical thicknesses and refractive index modulations. This analysis is to study possible deformations in the sinusoidal profile of the gratings due to shrinkage.

\subsection{Study of the shrinkage in the different materials}

In this case, the main idea is to show the maximum transmission efficiency (TE) and diffraction efficiency (DE) achieved for the holograms recorded on the different materials. For thick slanted gratings, it is difficult to obtain this information on real time due to the high angular selectivity and Bragg's condition detuning. Therefore, to obtain information of the DE at Bragg's condition, we have situated the readout laser at the angle where the maximum DE for long time recorded gratings is located to show the maximum DE achieved for these holograms.

The results for the different photopolymers are presented on Fig. 3, for the diffracted order situated around $3.7^{\circ}$ in air. For the PVA/AA based photopolymer, the value of DE is higher than $80 \%$, near the maximum achievable for this angle, showing the good response of this material to record slanted gratings and its viability in applications that require angular multiplexing, for example. For the $300 \mu \mathrm{m}$ thick layers of Biophotopol, it is difficult to measure the DEs at Bragg's angle. Very small deviations of this angle produce a huge change on the measured DE [27, 28]. In this case, to obtain the maximum $\mathrm{DE}$ as a function of time, we analysed many gratings recorded at different times getting their respective angular responses to obtain the maximum DE. The result shown is for the angle $7.2^{\circ}$ in air. In this case, the maximum $\mathrm{DE}$ is achieved after 30 seconds of exposition and it is near the one obtained for PVA/AA, higher than $80 \%$.

In the case of the HPDLC material, also high values, near $80 \%$ are obtained for an angle of $3.7^{\circ}$ in air. It is remarkable that in this case, the angle deviation does not affect significantly to the maximum value of DE. For these kind of materials, the shrinkage is reduced due to the PIPS effect [29] and the multifunctional monomer that causes compaction in the polymerized zones.

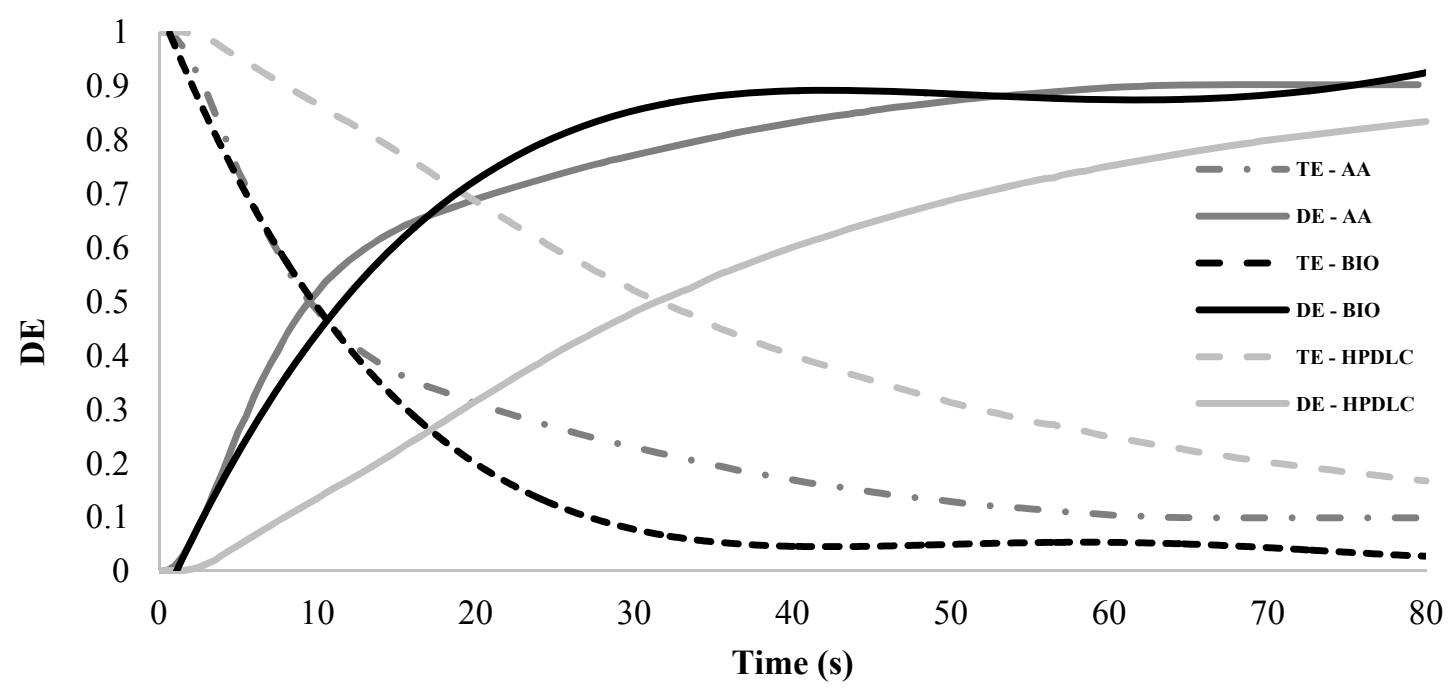

Fig. 3.DE as a function of recording time for the different photopolymers at Bragg's condition for diffracted order $+1: 75 \mu \mathrm{m} \mathrm{AA/PVA} \mathrm{material,} 300 \mu \mathrm{m}$ Biophotopol and $16 \mu \mathrm{m}$ HPDLC.

Once we have analysed the DE of each material, we measured the Bragg's angles of the orders +1 to obtain the value of $\mathrm{K}$ vector and check the possible variations of the $\mathrm{K}_{\mathrm{x}}$ component. We control the initial Bragg's angles in air for the diffracted orders, and we measured the new ones with an error of $\pm 0.002^{\circ}$. This accuracy give us an error of $0.09 \%$ in the determination of the shrinkage. Nevertheless, we must add additional imprecisions, firstly measuring the refractive index of the layer with an Abbe refractometer, $\mathrm{n}=1.4811 \pm 0.0005$, secondly, possible average changes in $\mathrm{n}$ of 0.003 due to polymerization, and thirdly, the repeatability that implies a total error in the shrinkage of $0.14 \%$. To measure the shrinkage, we followed the equation presented by Zhao [14], Eq. (2), obtaining the value of $\mathrm{K}$ from the angles of the 
both first orders \pm 1 . In Table 3, we present the measured angles in air for each photopolymer and their respective $\mathrm{K}_{\mathrm{x}}$ components. It is observable how in the AA/PVA the $\mathrm{K}_{\mathrm{x}}$ component decreases with long expositions, from $6.21 \mu \mathrm{m}^{-1}$ to $6.15 \mu \mathrm{m}^{-1}$ after 80 seconds of exposition. This drop of the $\mathrm{K}_{\mathrm{x}}$ value is due to an error determining the shrinkage with only one Bragg's angle, near $\pm 2 \%$, the same value of the highest values reported in this paper. In other words, the relative error, measuring only one Bragg's detuning angle, can be higher than $100 \%$. It is also appreciable how, even when the DE looks constant, the shrinkage continues growing for BIO and AA/PVA. For the HPDLC material, we observed a shrinkage close to the one of the AA/PVA and a very weak variation of the $\mathrm{K}_{\mathrm{x}}$ component.

Table 3. Measured shrinkage and $\mathrm{x}$ component of $\mathrm{K}$ vector for the three different photopolymers.

\begin{tabular}{|c|c|c|c|c|c|c|}
\hline $\begin{array}{c}\text { Recording } \\
\text { time (s) }\end{array}$ & $\begin{array}{c}\text { \% Shrinkage } \\
\mathbf{A A} \pm \mathbf{0 . 1 4}\end{array}$ & $\begin{array}{c}\text { Kx AA } \\
\left(\boldsymbol{\mu \mathbf { m } ^ { - \mathbf { 1 } } )}\right. \\
\mathbf{\pm 0 . 0 1}\end{array}$ & $\begin{array}{c}\mathbf{\%} \text { Shrinkage } \\
\mathbf{B I O} \pm \mathbf{0 . 2}\end{array}$ & $\begin{array}{c}\text { Kx BIO } \\
\left(\boldsymbol{\mu \mathbf { m } ^ { - 1 } )}\right. \\
\mathbf{\pm 0 . 0 1}\end{array}$ & $\begin{array}{c}\text { \% Shrinkage } \\
\text { HPDLC } \pm \mathbf{0 . 2}\end{array}$ & $\begin{array}{c}\text { Kx HPDLC } \\
\left(\boldsymbol{\mu m}^{-\mathbf{1}}\right) \pm \mathbf{0 . 0 1}\end{array}$ \\
\hline 5 & 0.05 & 6.21 & 0.8 & 7.34 & 0.4 & 6.19 \\
\hline 10 & 0.08 & 6.21 & 1.4 & 7.34 & 0.5 & 6.19 \\
\hline 20 & 0.05 & 6.19 & 2.6 & 7.37 & 0.8 & 6.19 \\
\hline 30 & 0.17 & 6.18 & 2.8 & 7.36 & 1.0 & 6.19 \\
\hline 40 & 0.29 & 6.16 & 3.2 & 7.37 & 1.2 & 6.20 \\
\hline 50 & 0.62 & 6.13 & 3.5 & 7.38 & 1.2 & 6.20 \\
\hline 80 & 1.31 & 6.13 & 3.5 & 7.40 & 1.2 & 6.20 \\
\hline
\end{tabular}

\subsection{Study of the diffracted orders}

It is interesting to use one of the multiple coupled wave theories to reproduce the angular scan of a holographic slanted grating to obtain information about different parameters such as the optical thickness or the refractive index modulation. These theories are also useful to study the deviation from the sinusoidal profile provided by the higher orders. In many of the shrinkage models reported [9-17], it is supposed that the sinusoidal profile remains after the shrinkage occurs. If there is any deviation from this profile during the shrinkage, it is supposed to be a variation in the higher diffracted orders. We have compared different coupled wave theories: Kogelnik wave theory $(\mathrm{KCW})$, rigorous coupled wave theory (RCW) and time-domain-difference-method (TDDM) comparing the approximate $\mathrm{KCW}$ model with the rigorous models.

For the slanted gratings analysed in this paper, only one of the third diffracted orders are inside of the Ewald's Sphere, nevertheless, in all the cases analysed its DE is weaker than $0.1 \%$ and in the most of them it is very difficult to detect and to fit it with the coupled wave theories.

For the different materials, we depicted the experimental angular scan around the +1 Bragg's condition. In Fig. 4 it is shown the comparison between the results for the PVA/AA material exposed during $40 \mathrm{~s}$ and the fitting simulations provided by the $\mathrm{KCW}$ and $\mathrm{RCW}$ models.

In the case of the Biophotopol material, the results of the fitting are shown in Fig. 5 we did not observe any higher diffraction order. It is also important to remark that, in this case, the RCW model is not suitable due to the large thicknesses of the material, that caused instabilities. In this case, to compare with the KCW, we used the FDTDM, observing good agreement between experimental data and both models. In this case, the max DE reached is reduced by the value of absorption and scattering coefficient around $13 \%$, due to the high thickness value.

For HPDLC materials, the RCW model is suitable to fit the angular response. This fitting is shown in Fig. 6. In this case, we can observe a deviation of the KCW model respect to the RCW due to the low thickness and high refractive index modulation. 


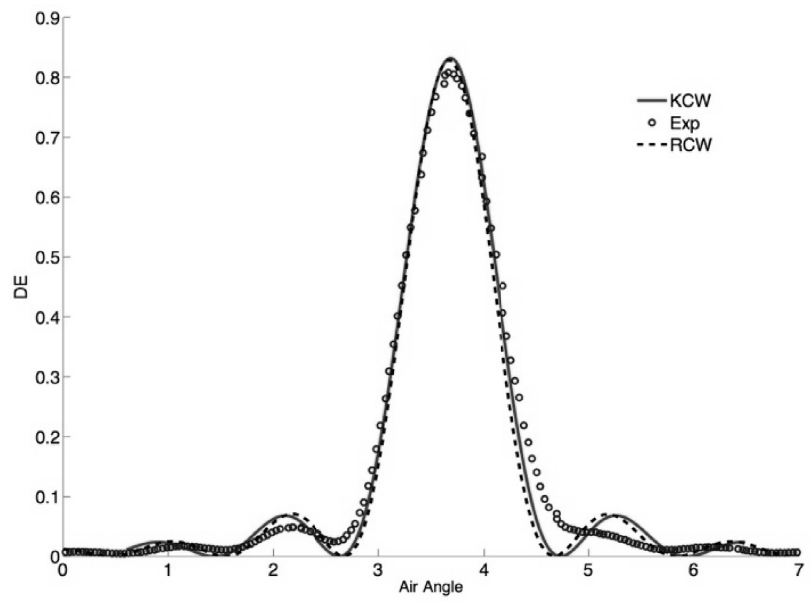

Figure 4. Angular response of diffracted order +1 for PVA/AA photopolymer and fittings obtained by KCW and RCW methods. The values obtained from the fitting are $\mathrm{d}=75 \mu \mathrm{m}, \mathrm{n}_{1}=0.00325, \alpha=0.0005 \mu \mathrm{m}^{-1}$

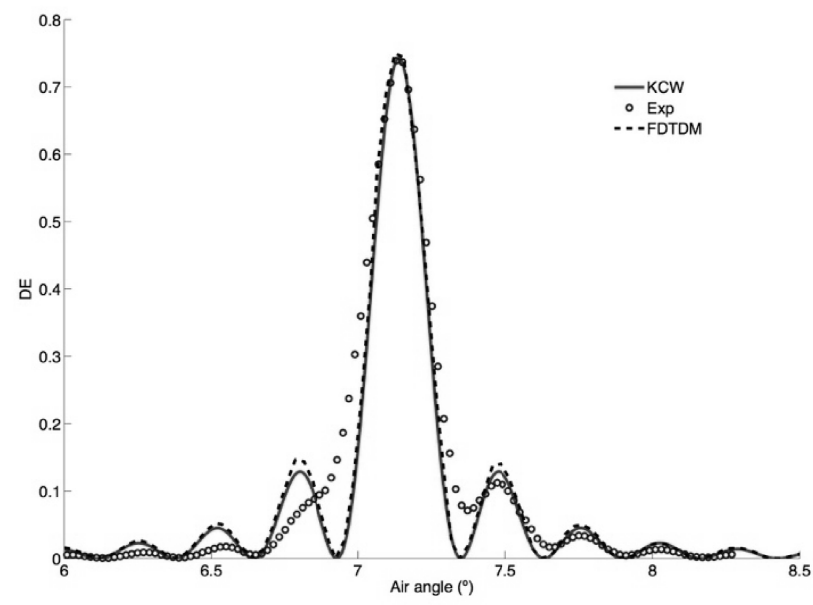

Figure 5. Angular response of diffracted order +1 for Biophotopol photopolymer and fittings obtained by KCW and FDTDM methods. The values obtained from the fitting are $\mathrm{d}=285 \mu \mathrm{m}, \mathrm{n}_{1}=0.0013, \alpha=0.0005 \mu \mathrm{m}^{-1}$

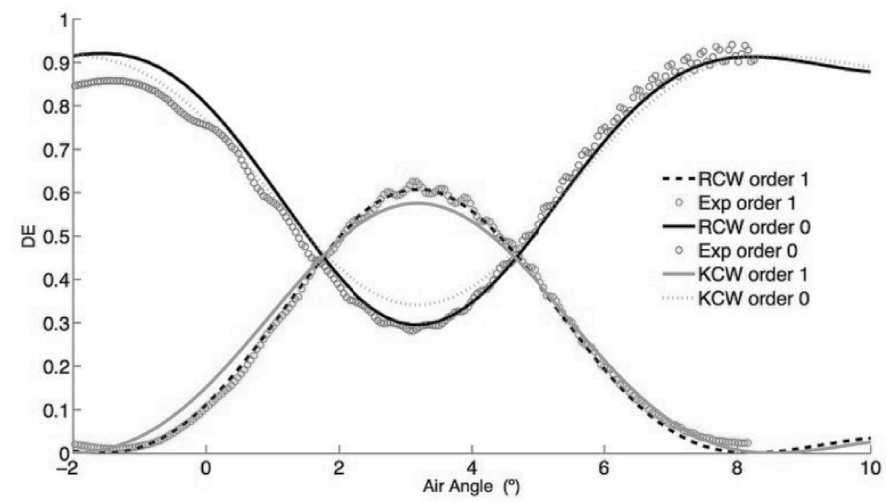

Figure 6. Angular response of diffracted order +1 for HPDLC photopolymer and fittings obtained by KCW and RCW methods. The values obtained from the fitting are $\mathrm{d}=16 \mu \mathrm{m}, \mathrm{n}_{1}=0.0115, \alpha=0.0006 \mu \mathrm{m}^{-1}$ 


\section{CONCLUSIONS}

In this work, we have compared different methods to study the dimensional changes in different families of photopolymers, measuring the holographic recording material shrinkage. The PVA/AA and Biophotopol materials have exhibited a variation of the $\mathrm{Kx}$ component of the grating vector. In this case, as it is not possible to measure the shrinkage by using the classical methods, the measurement of the Bragg's angles for both orders \pm 1 , from these data, we can calculate the shrinkage using two different equations with similar results.

For the third material, HPDLC, the value of $\mathrm{Kx}$ remains almost constant; nevertheless, for these metrology measurements, where high precision is required, we suggest also determining shrinkage from the values of both first Bragg's angles. To analyse how the shrinkage affects to the grating shape we have measured higher diffracted orders using different electromagnetic theories, we can conclude that the shrinkage does not increase the deformations in the sinusoidal profile significantly.

\section{Acknowledgments}

This work was supported by the "Ministerio de Economía y Competitividad" (projects FIS2015-66570-P and FIS201456100-C2-1-P) and by the "Generalitat Valenciana" of Spain (projects PROMETEOII/2015/015 and ISIC/2012/013).

\section{REFERENCES}

[1] M. S. Weiser, F. K. Bruder, T. Fäcke, D. Hönel, D. Jurbergs, and T. Rölle, "Self-processing, diffusion-based photopolymers for holographic applications," Macromol. Symp. 296(1), 133-137 (2010).

[2] J. Neumann, K. S. Wieking, D. Kip, "Direct laser writing of surface reliefs in dry, self-developing photopolymer films" Appl. Opt. 38, 5418-5421 (1999).

[3] X. T. Li, A. Natansohn, P. Rochon, "Photoinduced liquid crystal alignment based on a surface relief grating in an assembled cell," Appl. Phys. Lett 74, 3791-3793 (1999).

[4] C. P. Jisha, V. C. Kishore, Beena Mary John, V. C. Kuriakose, K. Porsezian, and C. Sudha Kartha, "Self-written waveguide in methylene blue sensitized poly (vinyl alcohol)/acrylamide photopolymer material," Appl. Opt. 47, 6502-6507 (2008).

[5] M. D. Lechner, "Photopolymers for optical memories and waveguides,". Electron. Prop. Polym. Relat. Compd. 63, 301-308 (1985).

[6] H. J. Coufal, D. Psaltis, G.T. Sincerbox, "Holographic Data Storage" Springer-Verlag: Berlin, Germany, (2000).

[7] M. Toishi, T. Takeda, K. Tanaka, T. Tanaka, A. Fukumoto, and K. Watanabe, "Two-dimensional simulation of holographic data storage medium for multiplexed recording," Opt. Express 16(4), 2829-2839 (2008).

[8] Y.-C. Su, C.-C. Chu, W.-T. Chang, and V. K. S. Hsiao, "Characterization of optically switchable holographic polymer-dispersed liquid crystal transmission gratings," Opt. Mater. 34(1), 251-255 (2011).

[9] P. Wang, B. Ihas, M. Schnoes, S. Quirin, D. Beal, S. Setthachayanon, T. Trentler, M. Cole, F. Askham, D. Michaels, S. Miller, A. Hill, W. Wilson, and L. Dhar, "Photopolymer media for holographic storage at $405 \mathrm{~nm}$," Proc. SPIE 5380, 283-288 (2004).

[10] J. T. Gallo and C. M. Verber, "Model for the effects of material shrinkage on volume holograms," Appl. Opt. 33(29), 6797-6804 (1994).

[11] M. P. Jordan and L. Solymar, “A note on volume holograms," Electron. Lett. 14(9), 271-272 (1978).

[12] J. T. Gallo and C. M. Verber, "Model for the effects of material shrinkage on volume holograms," Appl. Opt. 33(29), 6797-6804 (1994)

[13] J. H. Chen, D.-C. Su, and J.-C. Su, "Shrinkage- and refractive-index shift-corrected volume holograms for optical interconnects," Appl. Phys. Lett. 81(8), 1387-1389 (2002).

[14] C. Zhao, J. Liu, Z. Fu, and R. T. Chen, "Shrinkage correction of volume phase holograms for optical interconnects. Proc. SPIE 3005,” Optoelectronic Interconnects and Packaging IV, 224-229 (1997).

[15] G. Ramos, A. Álvarez-Herrero, T. Belenguer, F. del Monte, and D. Levy, "Shrinkage control in a photopolymerizable hybrid solgel material for holographic recording," Appl. Opt. 43(20), 4018-4024 (2004). 
[16] M. Moothanchery, I. Naydenova, and V. Toal, "Study of the shrinkage caused by holographic grating formation in acrylamide based photopolymer film," Opt. Express 19(14), 13395-13404 (2011).

[17] T. Sabel and M. Zschocher, "Imaging of Volume Phase Gratings in a Photosensitive Polymer, Recorded in Transmission and Reflection Geometry,” Appl. Sci. 4(1), 19-27 (2014).

[18] N. Pandey, I. Naydenova, S. Martin, and V. Toal, "Technique for characterization of dimensional changes in slanted holographic gratings by monitoring the angular selectivity profile," Opt. Lett. 33(17), 1981-1983 (2008).

[19] S. Gallego, A. Márquez, D. Méndez, C. Neipp, M. Ortuño, M. Alvarez, E. Fernandez, and A. Beléndez, "Realtime interferometric characterization of a polyvinyl alcohol based photopolymer at the zero-spatial frequency limit," Appl. Opt. 46(30), 7506-7512 (2007).

[20] M. Moothanchery, V. Bavigadda, V. Toal, and I. Naydenova, "Shrinkage during holographic recording in photopolymer films determined by holographic interferometry," Appl. Opt. 52(35), 8519-8527 (2013).

[21] M. Kawana, J. Takahashi, J. Guo, and Y. Tomita, "Measurement of polymerization-shrinkage evolution during curing in photopolymer with a white-light Fabry-Pérot interferometer," Opt. Express 23(12), 15356-15364 (2015)

[22] O'Neill, F.T., Lawrence, J.R., Sheridan, J.T., "Thickness variation of self-processing acrylamide-based photopolymer and reflection holography," Opt. Eng. 40(4), 533-539 (2001).

[23] O’Neill, F. T., Carr, A. J., Daniels, S. M., Gleeson, M. R., Kelly, J. V., Lawrence, J. R., \& Sheridan, J. T., "Refractive elements produced in photopolymer layers," Journal of Materials Science 40(15), 4129-4132 (2005).

[24] V. Navarro-Fuster, M. Ortuño, S. Gallego, A. Márquez, A. Beléndez, and I. Pascual, "Biophotopol's energetic sensitivity improved in $300 \mu \mathrm{m}$ layers by tuning the recording wavelength," Opt. Mater. 52, 111-115 (2016).

[25] S. Gallego, M. Ortuño, A. Márquez, R. Fernández, M. Álvarez, I. Pascual, A. Beléndez, "Influence of Thickness on the Holographic Parameters of H-PDLC Materials," International Journal of Polymer Science 2014, 528287 (2014).

[26]C. Neipp, A. Beléndez, S. Gallego, M. Ortuño, I. Pascual, J. Sheridan, and J. T. Sheridan, "Angular responses of the first and second diffracted orders in transmission diffraction grating recorded on photopolymer material," Opt. Express 11(16), 1835-1843 (2003).

[27] M. G. Moharam and T. K. Gaylord, "Rigorous coupled-wave analysis of planar-grating diffraction," J. Opt. Soc. Am. 71(7), 811-818 (1981).

[28] J. Francés, S. Bleda, C. Neipp, A. Márquez, I. Pascual, and A. Beléndez, "Performance analysis of the FDTD method applied to holographic volume gratings: Multi-core CPU versus GPU computing," Comput. Phys. Commun. 184(3), 469-479 (2013).

[29]Y. J. Liu and X. W. Sun, "Holographic polymer-dispersed liquid crystals: Materials, formation, and applications," Advances in OptoElectronics 2008, 684349 (2008) 